\title{
A New Classification for Cervical Ossification of the Pos- terior Longitudinal Ligament Based on the Coexistence of Segmental Disc Degeneration
}

\author{
Jun Ki Lee, Chang Hwa Ham, Woo-Keun Kwon, Hong Joo Moon, Joo Han Kim, Youn-Kwan Park \\ Department of Neurosurgery, Korea University Guro Hospital, Seoul, Korea
}

Objective : Classification systems for cervical ossification of the posterior longitudinal ligament (OPLL) have traditionally focused on the morphological characteristics of ossification. Although the classification describes many clinical features associated with the shape of the ossification, including the concept of spondylosis seems necessary because of the similarity in age distribution.

Methods : Patients diagnosed with OPLL who presented with increase signal intensity (ISI) on magnetic resonance imaging were surgically treated in our department. The patients were divided into two groups (pure versus degenerative) according to the presence of disc degeneration.

Results : Of 141 patients enrolled in this study, more than half (61\%) were classified into the degenerative group. The pure group showed a profound male predominance, early presentation of myelopathy, and a different predilection for ISI compared to the degenerative group. The mean canal compromise ratio (CC) of the ISI was $47 \%$ in the degenerative group versus $61 \%$ in the pure group $(p<0.0000)$. On the contrary, the global and segment motions were significantly larger in the degenerative group $(p<0.0000$ and $p=0.003$, respectively). The canal diameters and global angles did not differ between groups.

Conclusion : Classifying cervical OPLL based on the presence of combined disc degeneration is beneficial for understanding the disorder's behavior. CC appears to be the main factor in the development of myelopathy in the pure group, whereas additional dynamic factors appear to affect its development in the degenerative group.

Key Words : Classification · Intervertebral disc degeneration · Motion · Myelopathy · Ossification of the posterior longitudinal ligaments . Spinal canal.

\section{INTRODUCTION}

Ossification of the posterior longitudinal ligament (OPLL) is a disease in which ectopic bone formation occurs in the ligament due to hyperostotic changes ${ }^{11)}$. Progression of OPLL sometimes leads to neural compression, which can cause seri- ous symptoms or signs such as myelopathy and radiculopathy that require clinician attention. The pathogenesis of OPLL is multifactorial and involves both genetic and environmental factors, but it largely remains unclear ${ }^{6,17,18)}$.

Like other musculoskeletal systems, the cervical spine experiences local biological stress and acquires degenerative chang-

- Received : March 16, 2020 •Accepted : April 9, 2020

- Address for reprints : Youn-Kwan Park

Department of Neurosurgery, Korea University Guro Hospital, 148 Gurodong-ro, Guro-gu, Seoul 08308, Korea

Tel : +82-2-2626-3095, Fax : +82-2-863-1684, E-mail : ykapa76@yahoo.co.kr, ORCID : https://orcid.org/0000-0003-0501-3540

This is an Open Access article distributed under the terms of the Creative Commons Attribution Non-Commercial License (http://creativecommons.org/licenses/by-nc/4.0) which permits unrestricted non-commercial use, distribution, and reproduction in any medium, provided the original work is properly cited. 
es with aging. These changes are frequently comorbid in OPLL patients since the predilection of age and site are similar in both conditions. There is no doubt that the accompanying spondylosis and combined degeneration will probably affect the course of disease progression in various ways, but no such study has reported on this to date. Although numerous classification systems exist, most focus on the morphological characteristics of ligament ossification and show limited ability in describe the behavior and predict the progression of the disorder. Thus, this study aimed to determine whether comorbid segmental disc degeneration affects the disorder's progression and behavior. Furthermore, we suggest a new perspective on the classification of OPLL.

\section{MATERIALS AND METHODS}

This study was approved by the Institutional Review Board (K2020-0564-001). To evaluate the characteristics of our new classification method, we retrospectively reviewed the charts and radiographs of patients treated in our hospital.

\section{Patient population}

Between September 2009 and August 2019, a total of 216 patients who were diagnosed with cervical OPLL with myelopathy and underwent various decompressive surgeries with or without fusion in our department were retrospectively reviewed. The inclusion criteria were radiological confirmation of OPLL by 2-dimensional (2D) computed tomography (CT) and the presence of myelopathy on magnetic resonance imaging (MRI), such as increase signal intensity (ISI), with corresponding clinical symptoms and neurological signs. Patients were excluded if surgery was previously performed on the cervical spine $(n=6)$. Patients who visited the emergency room with trauma-induced myelopathy ( $\mathrm{n}=46$ ), or who showed ISI in the segment other than OPLL $(n=23)$ were also excluded. Finally, 141 patients were enrolled. Anterior discectomy/corpectomy and fusion was done in 13 patients. Laminoplasty was done in 44 patients. Remaining 84 patients underwent laminoplasty with posterior instrumented fusion.

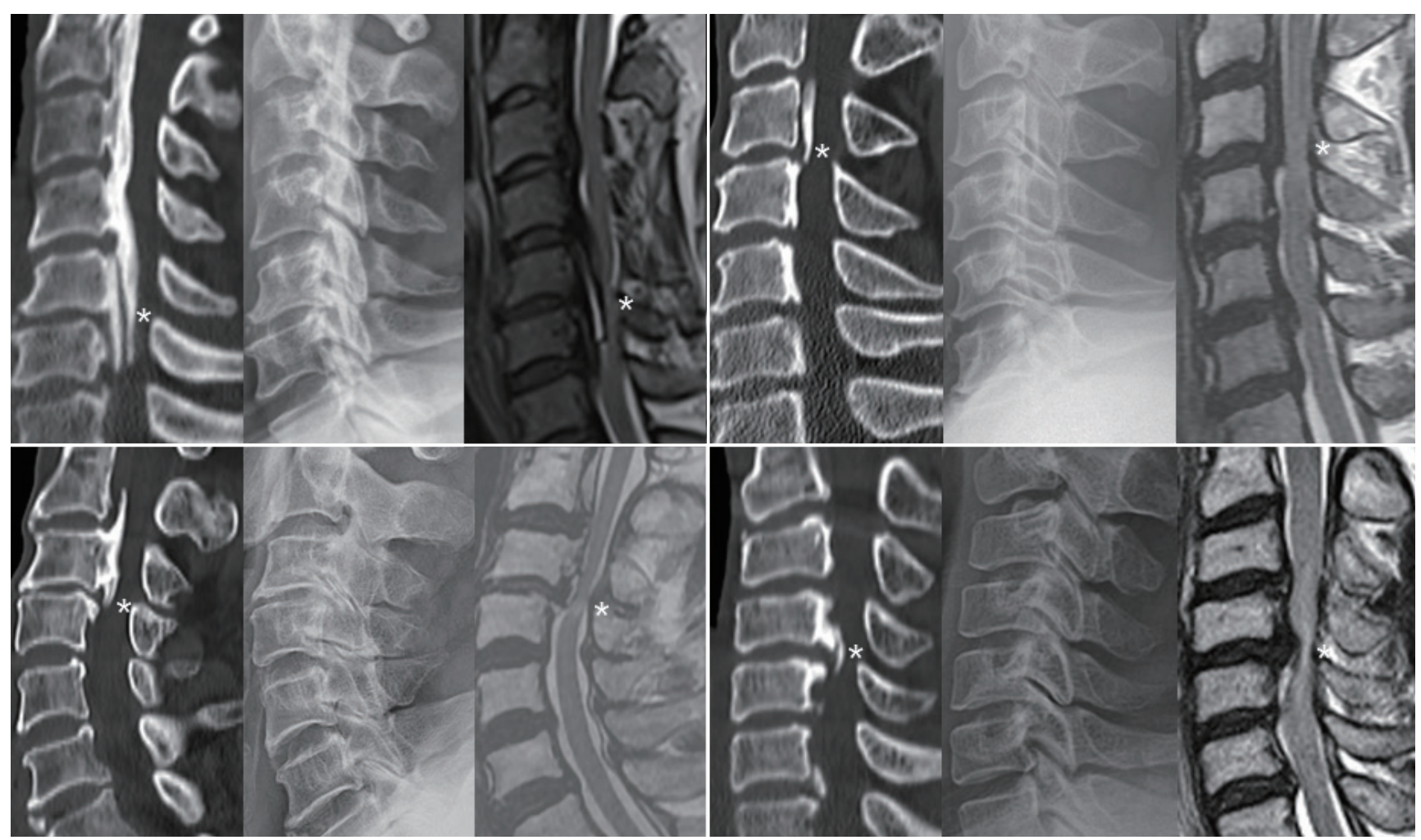

Fig. 1. Four representative cases showing the features of degenerative ossification of the posterior longitudinal ligament, including degenerative narrowing at the segment presenting myelopathy (asterisks) in mid-sagittal 2-dimensional reconstruction computed tomography image (left), lateral radiograph (middle), and sagittal T2-wighted magnetic resonance imaging (right). 


\section{Radiographic assessments}

Radiographic measurements were performed using the Patient Archiving Communication System software (PiViewSTAR, Seoul, Korea). Disc height and Cobb angle were measured in the neutral position on the lateral view of the preoperative cervical spine. Global and segmental ranges of motion (ROM) were measured on dynamic lateral radiographs. The anteroposterior diameter (APD) and canal compromise ratio (CC) of the level presenting ISI (index level) on MRI were measured in sagittal images of 2D CT at the plane parallel to the end plates of the vertebrae.

We classified the type of OPLL into two groups (pure and degenerative) based on the degenerative changes in the disc of index level using simple lateral radiographs. Disc height was measured along a straight line passing through the central points of the adjacent vertebral bodies. A disc height less than $10 \%$ lower than the reference disc height (usually C2/C3 disc) was considered normal, a 10-20\% reduction was deemed mild degeneration, and a more than $20 \%$ reduction was deemed severe $^{16,19)}$. The reference disc was changed occasionally to the tallest one in the cervical spine if the $\mathrm{C} 2 / \mathrm{C} 3$ disc was degener-

Table 1. Demographics and results of measurements of the patients with single ISI

\begin{tabular}{|c|c|c|c|}
\hline & \multicolumn{2}{|c|}{ Group } & \multirow{2}{*}{$p$-value } \\
\hline & Pure & Degenerative & \\
\hline Number of patients & 48 (39.0) & 74 (61.0) & \\
\hline Age, mean (years) & 57 & 61 & $0.007^{*}$ \\
\hline Sex, male : female & $4.1: 1$ & $2.6: 1$ & $0.0000^{*}$ \\
\hline JOA score, mean & 12.9 & 12.3 & 0.2 \\
\hline \multicolumn{4}{|l|}{ C2-C7 level } \\
\hline Lordosis $\left({ }^{\circ}\right)$ & 12 & 12 & 0.5 \\
\hline Motion $\left({ }^{\circ}\right)$ & 29 & 39 & $0.0000^{*}$ \\
\hline \multicolumn{4}{|l|}{ ISI level } \\
\hline Motion $\left({ }^{\circ}\right)$ & 4.7 & 6.1 & $0.003^{*}$ \\
\hline AP diameter (mm) & 10.8 & 10.6 & 0.18 \\
\hline Canal compromise (\%) & 61 & 47 & $0.0000^{*}$ \\
\hline
\end{tabular}

Values are presented as number (\%) unless otherwise indicated. *Indicates statistical significance. ISI : increased signal intensity, JOA : Japanese Orthopedic Association, AP : antero-posterior

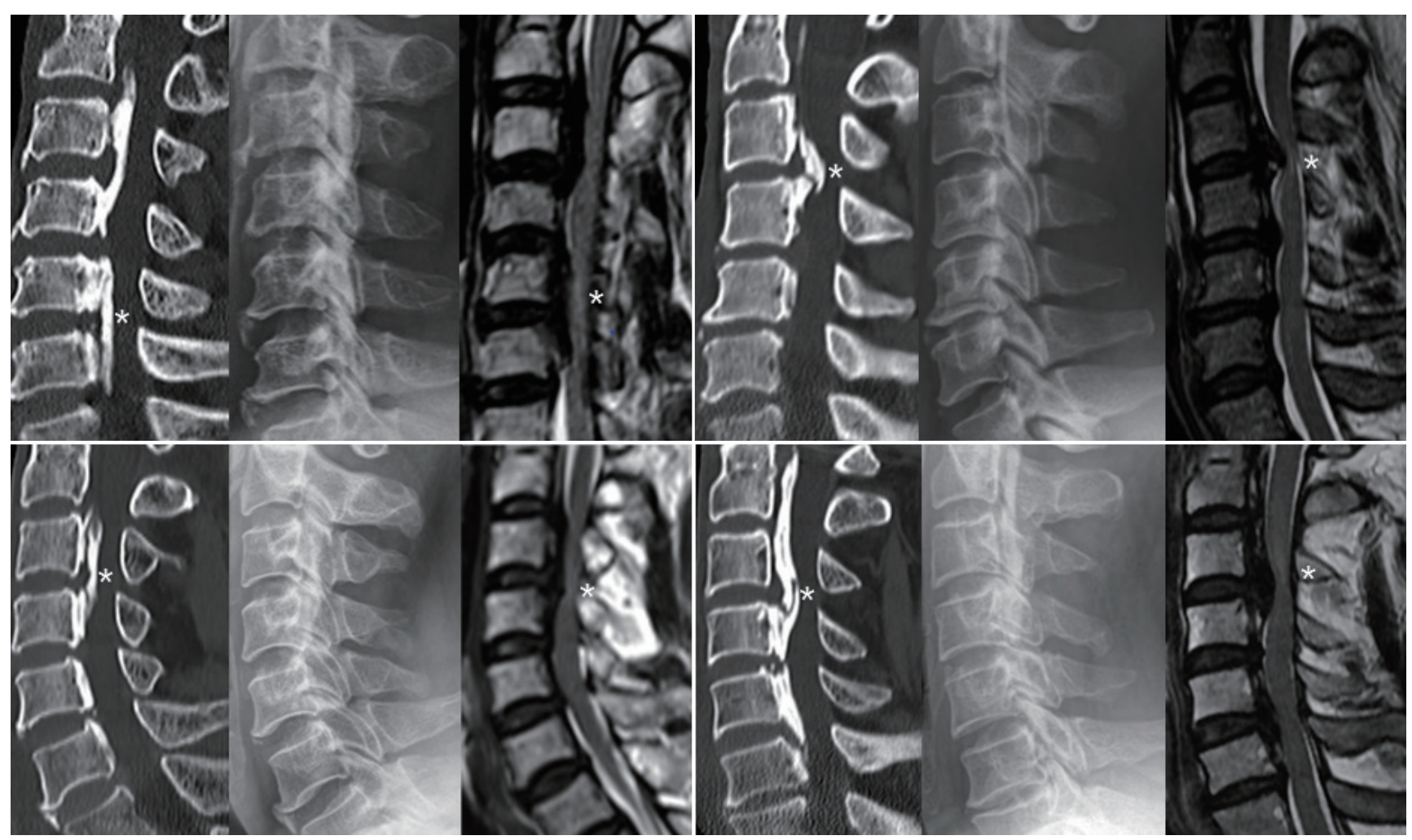

Fig. 2. Four representative cases showing the features of pure ossification of the posterior longitudinal ligament, including well preserved disc height at the segment presenting myelopathy (asterisks) in mid-sagittal 2-dimensional reconstruction computed tomography image (left), lateral radiograph (middle), and sagittal T2-wighted magnetic resonance imaging (right). 
ated. Radiological evidence of segmental degeneration was investigated by three reviewers (two neurosurgeons and one neurosurgical PGY4 resident) and defined by consensus of two or more thereof. OPLL types were also classified as segmental, continuous, mixed, and localized according to the Japanese Investigation Committee for Ossification of the Spinal Ligament criteria ${ }^{21)}$.

\section{Statistics}

All data management and statistical analyses were performed using the Analyse-it software (Analyse-it Ltd., Leeds, UK). The parametric statistical analysis was performed using Student's t test. Categorical variables were analyzed using the chi-square test. Significance was accepted at $p<0.05$.

\section{RESULTS}

Combined disc degeneration in the segment presenting with myelopathy was noted in $74(61 \%)$ of 122 patients with a single ISI (Fig. 1). The remaining 48 patients with normal disc height were classified as the pure group (Fig. 2). There was a strong male predominance in the pure group $(p<0.0000)$. The age of presenting myelopathy was slightly younger in the pure group than in the degenerative group $(p=0.007)$ (Table 1). The proportion of the degenerative group increased in an age-dependent manner, from $50 \%$ in the 40 s to $80 \%$ in the 70 s (Fig. 3). OPLL types differed between the groups. More long-segment types (mixed and continuous) were included in the pure group, while more short-segment types (segmental and localized) were included in the degenerative group (Fig. 4). The predilection of ISI also differed between the groups, located
(A)

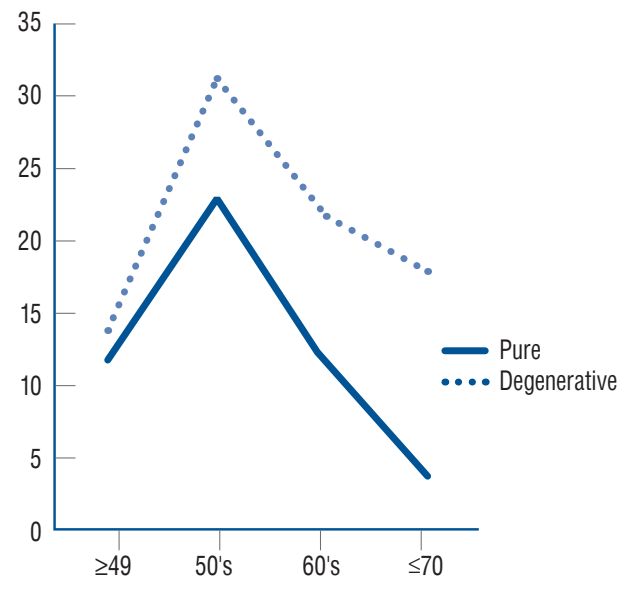

(B)

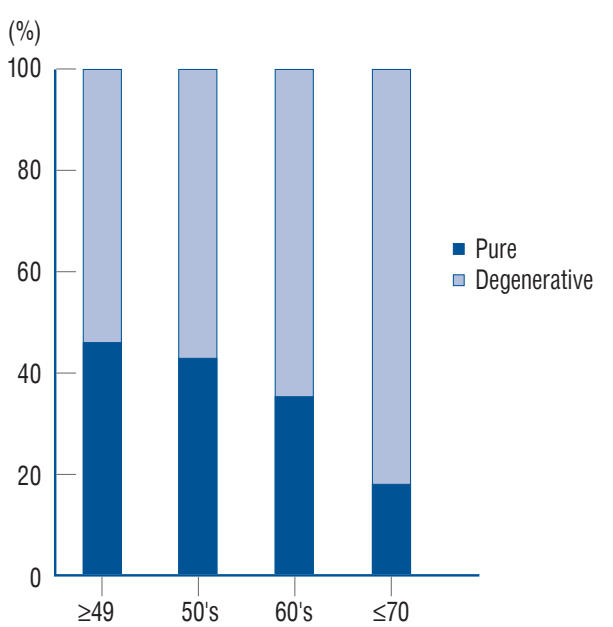

Fig. 3. Number of patients ( $y$ axis) in each group by age ( $x$ axis) (A). Cumulative graph showing the proportion of each group (B).

(A)

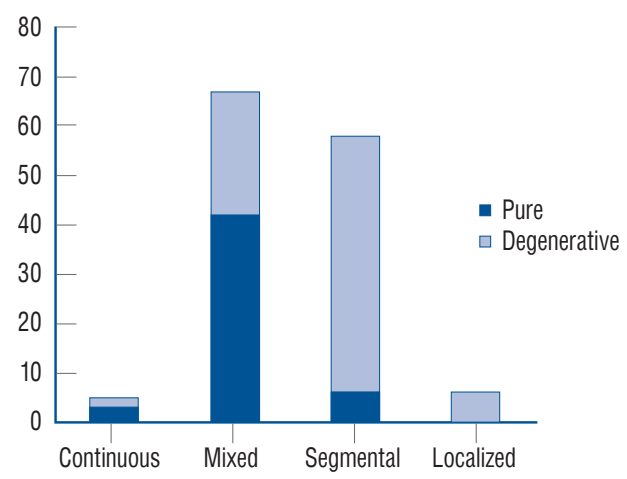

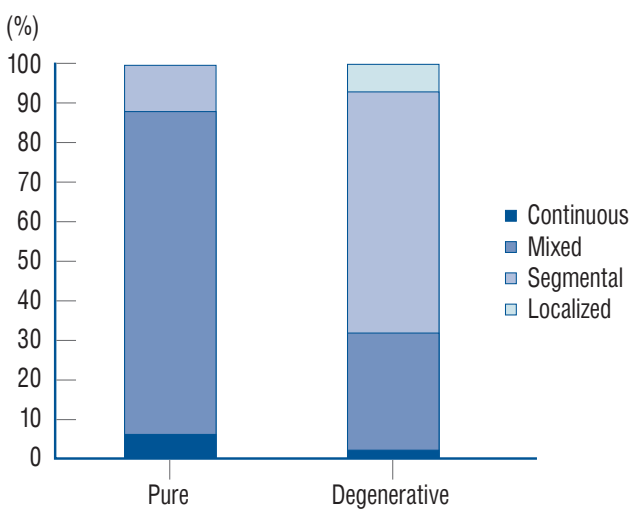

Fig. 4. Graph showing the number of patients (y axis) of each group according to the classification of the Japanese Ministry of Public Health and Welfare ( $x$ axis) (A). Cumulative graph showing the proportion of each group (B). 
rostrally at the $\mathrm{C} 3 / \mathrm{C} 4$ level in the pure group and caudally at the C5/C6 level in the degenerative group (Fig. 5).

The CC of the degenerative group was significantly lower

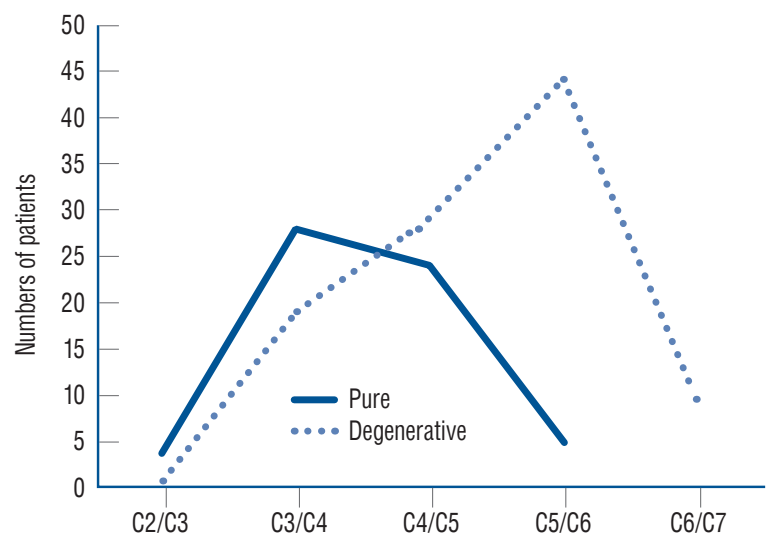

Fig. 5. Graph showing the number of patients (y axis) and the level of myelopathy ( $x$ axis) of each group.

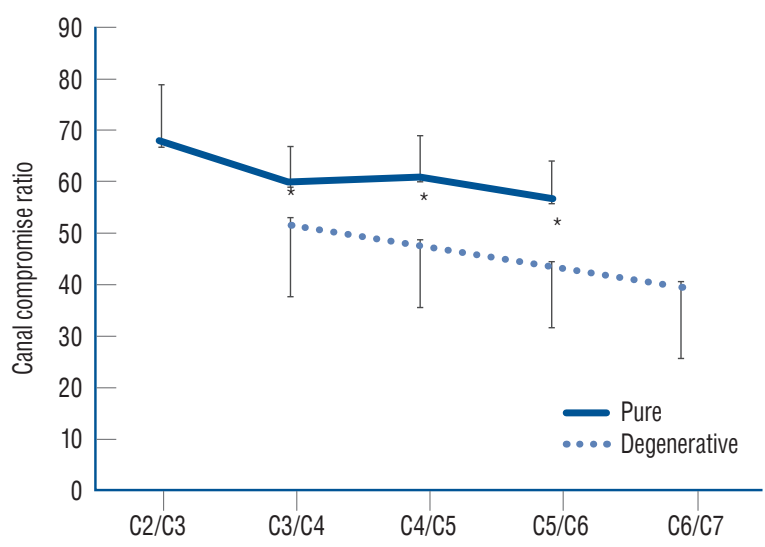

Fig. 6. Mean canal compromise ratio by level. Each canal compromise was measured in the level presenting myelopathy, which was significantly low in the degenerative group. ${ }^{*} p<0.01$. than that of the pure group (47\% and $61 \%$, respectively, $p<0.0000$ ), indicating that some other factors were exerting influence on the development of myelopathy in the degenerative group. The CC was high in the higher cervical vertebrae and low in the lower cervical vertebrae, indicating that myelopathy is prone to develop in areas of low CC in the lower cervical area, regardless of the group (Fig. 6). The global motion was significantly larger in the degenerative group $(p<0.0000)$, and there was a tendency toward an increase in global motion when the ISI was in the lower cervical area in both groups (Fig. 7). The segmental motion was also larger in the degenerative group, but it was small in amount $(p=0.003)$, and the tendency toward an increase in motion in the lower cervical area was noted only in the pure group. Neither the canal diameters nor the global angles differed between groups.

Nineteen patients in this study showed multiple ISI (Fig. 8). Among them, 11 were classified into the degenerative group, three in the pure group, and the remaining five in a mixed group. Because of the small numbers, the differences of all parameters with the patients with a single ISI were not significant, but the canal diameter was significantly narrower and the mean Japanese Orthopedic Association score was significantly worse among patients with multiple ISI (Table 2).

\section{DISCUSSION}

Although OPLL differs widely with respect to its shape, extent, and distribution, appropriate classification is critical since there may be significant variations in neurological presentation, disease progression, and risk of complications across OPLL
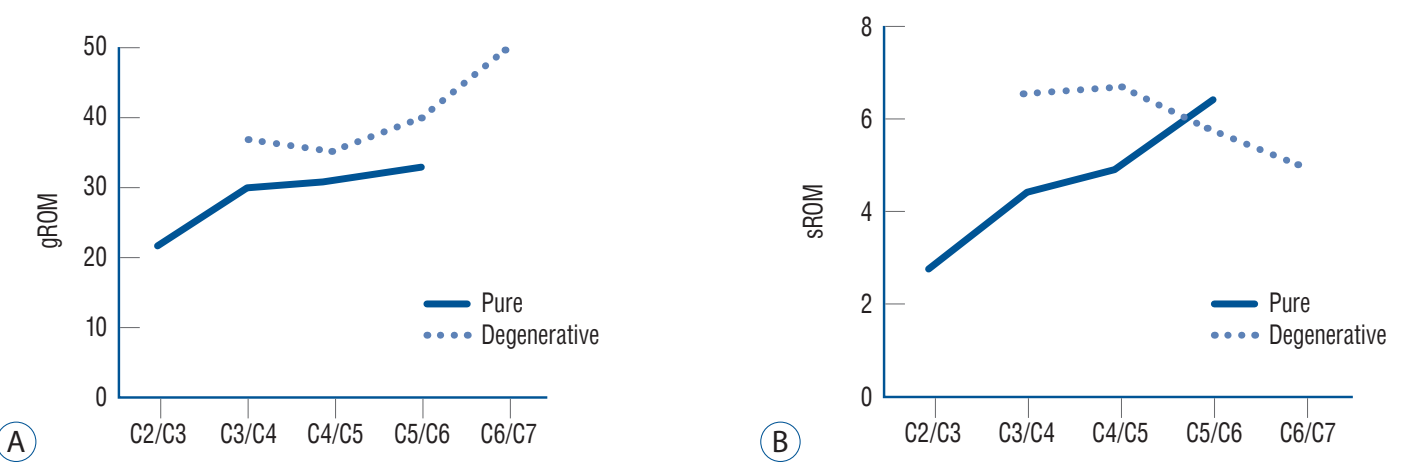

Fig. 7. Cervical range of motion according to the level presenting myelopathy. Mean values of global range of motion (gROM) (A), mean values of segmental range of motion (SROM) (B). 

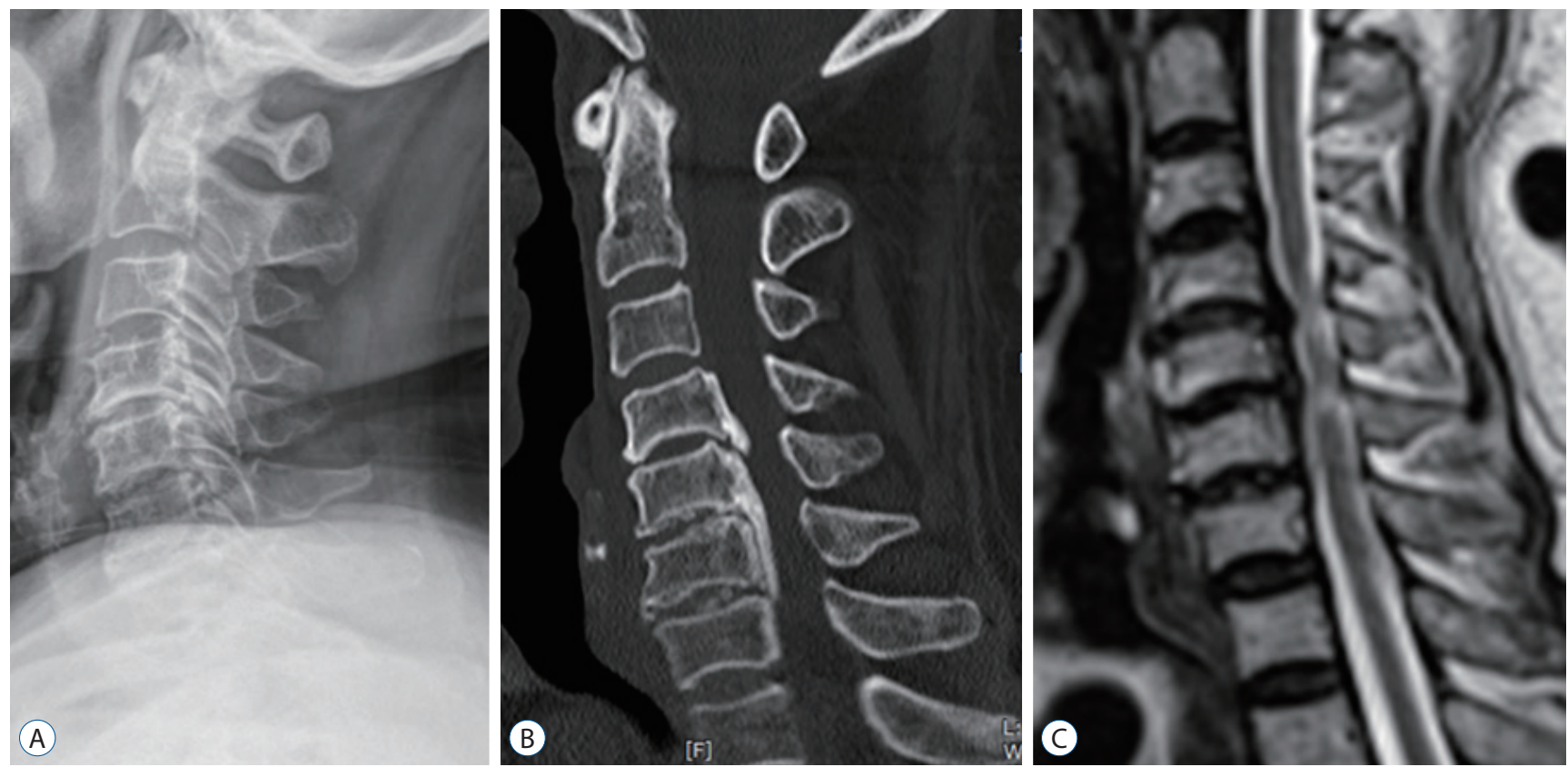

Fig. 8. Lateral radiograph (A), sagittal 2-dimensional reconstruction computed tomography image (B), and sagittal T2-weighted magnetic resonance imaging (C) of 76-year-old female patient with multiple increase signal intensity at $\mathrm{C} 4 / \mathrm{C} 5$ and $\mathrm{C} 5 / \mathrm{C} 6$, showing the features of degenerative type, including low compromise ratio (35-40\%) and preserved global motion $\left(56^{\circ}\right)$.

Table 2. Comparison of patients with single versus multiple increased signal intensities

\begin{tabular}{lccl}
\hline & \multicolumn{2}{c}{ Increased signal intensity } & \\
\cline { 2 - 3 } & Single & Multiple & -value \\
\hline Number of patients & 122 & 19 & \\
Age, mean (years) & 58 & 66 & 0.45 \\
\hline JOA score & 12.8 & 10.5 & $0.0084^{*}$ \\
Canal compromise $(\%)$ & 53 & 50 & 0.1 \\
Global motion $\left({ }^{\circ}\right)$ & 36 & 36 & 0.48 \\
\hline AP diameter $(\mathrm{mm})$ & 10.7 & 9.8 & $0.0000^{*}$ \\
\hline
\end{tabular}

*Indicates statistical significance. JOA : Japanese Orthopedic Association, AP : antero-posterior

subtypes. While numerous classification systems have been suggested $^{20)}$, to our knowledge, no system can properly characterize and best categorize its subtypes. Most classification systems for cervical OPLL are based on morphological characteristics, including the overall shape of the ossification of the ligament ${ }^{20)}$. On the contrary, we focused on the segment of myelopathy developed, looked for changes superimposed on the OPLL and found as many degenerative changes as in the normal populations. Disc degeneration is generally observed in $25 \%$ of patients younger than 40 years of age, $50 \%$ of those over the age of 40 years, and $85 \%$ of those over the age of 60 years ${ }^{8)}$. This picture was completely replicated in OPLL patients in this study (Fig. 3). While the predilection of the level presenting ISI in pure OPLL was in the C3/C4 level, superimposition of degenerative changes moved the myelopathy site down to a lower level, where degenerative changes usually occur in the general population. This is a solid evidence of the influence of combined degeneration on the behavior of OPLL disorders (Fig. 5).

Static compression has been proven to be the single most important risk factor for the development of myelopathy ${ }^{5,13)}$. However, a consensus on the involvement of dynamic factors has not yet been established. Matsunaga found a certain point $(60 \%)$ of compression at which all patients above that point became myelopathic ${ }^{12}$. The patients below that point were either myelopathic or not. Interestingly, the compression ratios did not differ between groups, but the ranges of motion were significantly different. Later, he replicated this finding with a multi-center study ${ }^{10)}$ and suggested that dynamic factors may be largely involved in inducing myelopathy. However, recent papers have failed to reproduce this finding ${ }^{2,4)}$. This may be due to the small cohorts and the inherent nature of the data source. It is generally believed that myelopathy develops primarily due to compression from the ossified ligament; however, some patients with smaller ossifications exhibit myelopa- 
thy, whereas others with marked ossification do not. This appeared as a wide range of compression ratios in myelopathic patients in a previous series. For example, in Matsunaga's study, the range of space available for the spinal cord (SAC) calculated based on plain X-ray images of myelopathic patients was between 4.5 and $14 \mathrm{~mm}^{9}$. The standard deviation (SD) of the mean compression ratio measured in CT in recent studies were also high-16\% in Chang et al.'s series ${ }^{2)}$ (mean, $50[n=34]$ ) and $13 \%$ in Du et al.'s study) (mean, 40 [n=54]). Similarly, the CC ratio of our pre-classified cohort also varied from $25 \%$ to $87 \%$ (mean, 53 [n=121]; SD, 14). Thus, static factors alone cannot account for the pathogenesis of myelopathy, and the variation must have other causes. Any responsible factors must primarily be dynamic. However, data-source heterogeneity results in even greater variation. From this point of view, a classification system that can reduce the heterogeneity of OPLL will increase our understanding of the disorder's behavior.

As shown in this study, the superimposition of degenerative changes is one controllable source of heterogeneity. The SD of the CC ratio in the pure group was decreased to $9 \%$ (range, 87-37; mean, 61). Unfortunately, the SD in the degenerative group remained at $13 \%$ (range, 76-25; mean, 47), implying that other factors including dynamic factors are still substantially active. There are other potential sources of OPLL heterogeneity. More than a dozen potentially related genes have been identified; each one has its own site of action and manner of expression. Subgrouping of the patients based on genetic studies looks ideal, but it cannot be accomplished within a year.

Although OPLL is a genetically linked disorder, environmental factors play a role in its development. The interaction between them looks mutually competitive. If a patient has a strong genetic background, the ossification starts earlier in their life and becomes thicker, and the motion reduces from the resulting stiffness; therefore, there is a lower chance of segmental degeneration and the ossification presents in its pure form. If a patient has a weak background, the ossification starts a little later, and the segment thickness and stiffness are not very high. Thus, they have a greater chance of developing degeneration. The continuous and mixed OPLL types were often associated with more severe spinal canal stenosis, smaller space available for the SAC, and higher compression ratios compared to the segmental and localized types ${ }^{14,20)}$. Concordantly, more patients with the long-segment type (continuous and mixed) belonged to the pure group, while more patients with the short-segment type (segmental and localized) belonged to the degenerative group in this study (Fig. 4).

$\mathrm{ROM}$ of the cervical spine is known to decrease as age increases. The C2-C7 motion of healthy Asian adults was reportedly 59 degrees in their 20s, 52 degrees in their 40s, and 45 degrees in their $60 \mathrm{~s}^{15}$. In the meantime, if OPLL develops and progresses, the spinal motion further decreases pathologically due to ligament ossification ${ }^{7)}$. Hence, the mean global ROM in this study was 29 degrees in the pure group and 39 degrees in the degenerativegroup, which roughly corresponds to $60 \%$ and $81 \%$ of the same age general population, respectively. The age-related reduction of the segmental ROM in the general population mostly occurs in two lower (C5/C6, C6/ C7) and one upper (C1/C2) level, which are more mobile in younger individuals. However, the segmental ROM of the three middle (C2/C3 to $\mathrm{C} 4 / \mathrm{C} 5)$ vertebrae usually remains unchanged. Thus, 40 years of age and above, it is the lowest at C2/C3 (9 degrees) and the highest at C5/C6 (14 degrees) ${ }^{15}$. The segmental ROM is known to be negatively correlated with the CC ratio in OPLL ${ }^{5}$. The mean segmental ROM of each group measured in this study was 4.7 degrees in the pure group and 6.1 degrees in the degenerativegroup, which roughly correspond to $35 \%$ and $47 \%$ of the same age population, respectively. As a result, the global ROM is preserved twice more than segmental ROM in OPLL disorders. This is one potential reason for the strong involvement of global ROM as a dynamic factor.

There was no intergroup difference in the APD among patients with a single ISI. But the values were only $10.7 \mathrm{~mm}$, significantly lower than that of the normal Asian population $(12.5-13.5 \mathrm{~mm})^{3}$. Moreover, the mean APD of multiple ISI patients was significantly lower than that of single ISI patients (Table 2). This suggests that a narrow APD, a so-called developmental stenosis, also plays an important role in the development of spondylotic myelopathy ${ }^{1)}$ and OPLL ${ }^{14)}$, especially in severe multifocal myelopathy.

This study confirmed that the coexistence of spondylotic changes altered the progression and behavior of OPLL. Since there were some clear intergroup differences, the whole picture of OPLL looked heterogenous. This result suggests that this classification may be helpful for selecting high-risk patients and determining treatment plans in OPLL patients without myelopathy. For example, asymptomatic OPLL pa- 
tients with a CC less than 50\% may require clinical attention when the maximum cord compression is located in the lower cervical area and degenerative changes are combined but the global motion is preserved. Based on our concept, further prospective studies and comparative studies in healthy people could lead to development of better therapeutic guidelines.

There are several limitations to this study related to the inherent nature of the data used. At first, ISI on MRI was used as a basis for the development of myelopathy and CC ratio was used as a static factor for OPLL. However, the occurrence of ISI and the time of radiologic examination were not simultaneous but were inevitably somewhat delayed. Therefore, it cannot be said that $\mathrm{CC}$ is a value at the time when myelopathy is expressed. Second, although CC ratio is the main static factor in OPLL, the coexisting degenerative changes in the surrounding tissues including disc space height loss can also be another factor, but this was not included in this study due to measurement difficulty and lack of standardization. Lastly, it is necessary to develop a strict methodology for the classification on the severity of disc degeneration for further validation. Nevertheless, this study provides new knowledge in many ways.

\section{CONCLUSION}

The classification of OPLL by the presence of combined disc degeneration is simple to apply and is beneficial for understanding the disorder's progression and behavior. The CC is the main factor in the development of myelopathy in pure OPLL, whereas dynamic factors seem to exert an influence on the development of myelopathy in relatively lesser canal compromise states in degenerative OPLL.

\section{CONFLICTS OF INTEREST}

No potential conflict of interest relevant to this article was reported.

\section{INFORMED CONSENT}

This type of study does not require informed consent.

\section{AUTHOR CONTRIBUTIONS}

\author{
Conceptualization : YKP \\ Data curation : HJM, JHK, YKP \\ Formal analysis : JKL, CHH, YKP \\ Methodology : JKL, CHH, YKP \\ Project administration : HJM \\ Visualization : JKL, YKP \\ Writing - original draft : JKL, YKP \\ Writing - review \& editing : JKL, WKK, JHK, YKP
}

\section{ORCID}

$\begin{array}{ll}\text { Jun Ki Lee } & \text { https://orcid.org/0000-0003-3507-3237 } \\ \text { Chang Hwa Ham } & \text { https://orcid.org/0000-0003-1020-4210 } \\ \text { Woo-Keun Kwon } & \text { https://orcid.org/0000-0003-0432-8620 } \\ \text { Hong Joo Moon } & \text { https://orcid.org/0000-0002-3841-9080 } \\ \text { Joo Han Kim } & \text { https://orcid.org/0000-0002-4747-9763 } \\ \text { Youn-Kwan Park } & \text { https://orcid.org/0000-0003-0501-3540 }\end{array}$

\section{- Acknowledgements}

This article was presented in ASIA Spine 2019, Seoul, Korea. We would like to thank Editage (www.editage.co.kr) for English language editing.

\section{References}

1. Bednarik J, Kadanka Z, Dusek L, Kerkovsky M, Vohanka S, Novotny O, et al. : Presymptomatic spondylotic cervical myelopathy: an updated predictive model. Eur Spine J 17 : 421-431, 2008

2. Chang H, Song KJ, Kim HY, Choi BW : Factors related to the development of myelopathy in patients with cervical ossification of the posterior longitudinal ligament. J Bone Joint Surg Br 94 : 946-949, 2012

3. Chazono M, Tanaka T, Kumagae Y, Sai T, Marumo K : Ethnic differences in pedicle and bony spinal canal dimensions calculated from computed tomography of the cervical spine: a review of the English-language literature. Eur Spine J 21 : 1451-1458, 2012

4. Du YQ, Duan WR, Chen Z, Wu H, Jian FZ : Risk factors and management of dural defects in anterior surgery for cervical ossification of the posterior longitudinal ligament. World Neurosurg 111 : e527-e538, 2018

5. Fujiyoshi T, Yamazaki M, Okawa A, Kawabe J, Hayashi K, Endo T, et al. : Static versus dynamic factors for the development of myelopathy in patients with cervical ossification of the posterior longitudinal ligament. J 
Clin Neurosci 17 : 320-324, 2010

6. Inamasu J, Guiot BH, Sachs DC : Ossification of the posterior longitudinal ligament: an update on its biology, epidemiology, and natural history. Neurosurgery 58 : 1027-1039; discussion 1027-1039, 2006

7. Ito K, Yukawa Y, Machino M, Kobayakawa A, Kato F : Range of motion determined by multidetector-row computed tomography in patients with cervical ossification of the posterior longitudinal ligament. Nagoya J Med Sci 77 : 221-228, 2015

8. Kelly JC, Groarke PJ, Butler JS, Poynton AR, O'Byrne JM : The natural history and clinical syndromes of degenerative cervical spondylosis. Adv Orthop 2012 : 393642, 2012

9. Matsunaga S, Kukita M, Hayashi K, Shinkura R, Koriyama C, Sakou T, et al. : Pathogenesis of myelopathy in patients with ossification of the posterior longitudinal ligament. J Neurosurg 96(2 Suppl) : 168-172, 2002

10. Matsunaga S, Nakamura K, Seichi A, Yokoyama T, Toh S, Ichimura S, et al. : Radiographic predictors for the development of myelopathy in patients with ossification of the posterior longitudinal ligament: a multicenter cohort study. Spine (Phila Pa 1976) 33 : 2648-2650, 2008

11. Matsunaga S, Sakou T : Ossification of the posterior longitudinal ligament of the cervical spine: etiology and natural history. Spine (Phila Pa 1976) 37 : E309-E314, 2012

12. Matsunaga S, Sakou T, Taketomi E, Komiya S: Clinical course of patients with ossification of the posterior longitudinal ligament: a minimum 10year cohort study. J Neurosurg 100(3 Suppl Spine) : 245-248, 2004

13. Morio Y, Nagashima H, Teshima R, Nawata K : Radiological pathogen- esis of cervical myelopathy in 60 consecutive patients with cervical ossification of the posterior longitudinal ligament. Spinal Cord 37 : 853857, 1999

14. Onishi E, Sakamoto A, Murata S, Matsushita M : Risk factors for acute cervical spinal cord injury associated with ossification of the posterior longitudinal ligament. Spine (Phila Pa 1976) 37 : 660-666, 2012

15. Park HJ, Jeon JS, Lee PE : Range of motion of cervical spine in normal Korean people. J Korean Soc Spine Surg 11 : 83-89, 2004

16. Park YK, Moon HJ, Kwon TH, Kim JH : Long-term outcomes following anterior foraminotomy for one- or two-level cervical radiculopathy. Eur Spine J 22 : 1489-1496, 2013

17. Saetia K, Cho D, Lee S, Kim DH, Kim SD : Ossification of the posterior longitudinal ligament: a review. Neurosurg Focus 30 : E1, 2011

18. Sakou T, Matsunaga S, Koga H : Recent progress in the study of pathogenesis of ossification of the posterior longitudinal ligament. J Orthop Sci 5 : 310-315, 2000

19. Sugawara T, Itoh Y, Hirano Y, Higashiyama N, Mizoi K : Long term outcome and adjacent disc degeneration after anterior cervical discectomy and fusion with titanium cylindrical cages. Acta Neurochir (Wien) 151 : 303-309; discussion 309, 2009

20. Tetreault L, Nakashima H, Kato S, Kryshtalskyj M, Nagoshi N, Nouri A, et al. : A systematic review of classification systems for cervical ossification of the posterior longitudinal ligament. Global Spine J 9 : 85-103, 2019

21. Tsuyama $N$ : Ossification of the posterior longitudinal ligament of the spine. Clin Orthop Relat Res (184) : 71-84, 1984 